\title{
Análise da Postura e dos Desconfortos de Gestantes atendidas pela Estratégia Saúde da Família
}

\author{
Fábia Maria de Santana ${ }^{1}$, Flávia Gomes da Silva ${ }^{2}$ Daniela Cavalcante e Silva Novais Carvalho ${ }^{1}$, \\ Dayanne Lays Bezerra Denoa ${ }^{3}$; Hellen Lúcia Cruz Caldas Lins ${ }^{4}$; Maria Eliana Pierre Martins ${ }^{5}$
}

\begin{abstract}
Resumo: Durante a gestação o organismo da mulher passa por diversas modificações hormonais e anatômicas que terá repercussão no sistema musculoesquelético. Essas modificações prévias podem causar algum dano ou agravar para um estado de complicação ao ciclo gravídico-puerperal. AO Estudo é descritivo, do tipo transversal e com análise quantitativa. A amostra foi composta por 20 gestantes, que tiveram seus dados coletados através de uma avaliação postura e entrevista, utilizando um questionário com informações sociodemográficas e sobre queixas e desconfortos durante a gestação. A pesquisa foi realizada na cidade de Cajazeiras- $\mathrm{Pb}$, com as gestantes com idades variando entre 16 e 39 anos. A lombalgia foi à causa mais evidenciada entre as participantes e dentre as alterações posturais mais encontradas foram a anteroversão pélvica, protusão de ombros e anteriorização da cabeça. A gestação é um processo fisiológico compreendido pela sequência de adaptações e alterações ocorridas no corpo da mulher a partir da fertilização. As mudanças que ocorrem no corpo feminino acontecem devido à quantidade excessiva de hormônios e alterações no sistema musculoesquelético, responsáveis pelas adaptações do organismo a sua nova condição. O estudo evidenciou que a lombalgia gestacional é a queixa mais freqüente em gestantes, visto que a maioria das participantes referiram sentir esta e outras alterações, o que demonstra que o período gestacional trás repercussões físicas importantes e que merecem atenções dos profissionais de saúde, uma vez que altera o bem estar das gestantes.
\end{abstract}

Palavras-chave: alterações posturais, gestantes, obstetrícia

\section{Postural analysis and discomforts of Pregnant attended by Family Health Strategy}

\begin{abstract}
During pregnancy a woman's body goes through many hormonal and anatomical changes that will impact on the musculoskeletal system. These changes may cause harm or serious complication of a state prior to pregnancy and childbirth. The sample consisted of 20 pregnant women who had their data collected in the form of interviews using a questionnaire on sociodemographic information and on complaints and discomforts during pregnancy. It was also made a postural assessment. It was confirmed discomfort and changes in the body of pregnant women according to the reported in the literature. Low back pain was the cause most evident among participants, set amidst them stood out to pelvic anteversion, protrusion of shoulders and forward head posture. Pregnancy is a physiological process understood by the following adaptations and changes in the woman's body from fertilization. The changes that occur in the female body happen due to excessive amount of hormones, the body responsible for adaptations to their new condition. Low back pain is considered a cause gestational common in pregnant women, as this study back pain in most cases, the participants reported feeling discomfort and pain being the cause of the low back pain. Postural assessment, postural changes were noted as not significant, but the present study found changes in the attitudes of pregnant women and they were the other prominent forward head posture, hyperlordosis protrusion of pelvic anteversion and shoulders.
\end{abstract}

Keyword: postural changes, pregnancy, obstetrics

\footnotetext{
${ }^{1}$ Mestranda, ciências da saúde, Faculdade de Medicina do ABC, Santo André, SP, Brasil. Email: fabiagarban@hotmail.com

${ }^{2}$ Fisioterapeuta, pela faculdade Santa Maria

${ }^{3}$ Biomedica, pela faculdade Leão Sampaio

${ }^{4}$ Médica. Mestre em Saúde da Criança e do Adolescente pela Universidade Estadual do Ceará. Doutoranda em Ciências da Saúde pela Faculdade de Medicina do ABC - São Paulo. E-mail: hellenmcruz@ hotmail.com;

${ }^{5}$ Médica. Doutoranda em Ciências da Saúde pela Faculdade de Medicina do ABC - São Paulo. E-mail: epierremartins@hotmail.com
} 


\section{Introdução}

A gestação impõe algumas modificações fisiológicas que ocorrem em todos os sistemas do corpo da mulher. Estas alterações decorrem, principalmente, de fatores hormonais e mecânicos podendo levar a vários sintomas e desconfortos durante a gestação, porém não é considerada uma condição patológica (SOARES et al, 2008).

As mudanças que ocorrem no organismo materno para o crescimento e desenvolvimento do feto estão relacionadas a grandes ajustes fisiológicos e anatômicos. Essas alterações ocorrem desde o início da gestação afetam o funcionamento de vários sistemas no corpo humano, principalmente o sistema musculoesquelético, sendo esse um processo de transformações e adaptações que em algumas mulheres trazem prejuízos que podem resultar em dor e limitações em suas atividades de vida diárias. (FREITAS, 2008).

As posturas incorretas adotadas durante a fase gestacional causam à coluna vertebral e as articulações esforço desnecessário. As alterações musculoesqueléticas ocorrem frequientemente, tais como: cervicalgia, dorsalgia, lombalgia e dor sacrilíaca. A incidência álgica na região lombar pode chegar a 50\%, enquanto que na coluna lombar chega a 80\% (MACHADO et al, 2011).

As alterações na biomecânica da postura das grávidas são respostas a vários fatores inerentes a essa fase, como o aumento dos seios, do útero gravídico, ganho de peso e instabilidade articular (MOREIRA et al, 2011). Essas adaptações são devido a perturbação das curvas fisiológicas da coluna pela maior inclinação anterior da pelve e rotação externa dos membros inferiores, que permitem a maior base de sustentação (SENHORINHO et al, 2003)

O objetivo da pesquisa foi identificar quais alterações posturais e desconfortos acometem as mulheres no período gestacional. Para assim poder desenvolver meios preventivos que minimizem ou evitem distúrbios e complicações provenientes destas alterações, como também, facilitar o desempenho das atividades de vida diária.

\section{Método}

Este estudo caracterizou-se por ser uma pesquisa descritiva do tipo transversal, com análise quantitativa, o mesmo seguiu os princípios em consideração os aspectos éticos contidos na resolução 196/96 do Conselho Nacional de Saúde (CNS) que regulamenta a pesquisa que envolve seres humanos (BRASIL, 1996). 
Todos os pacientes selecionados leram ou foram informados sobre o termo de consentimento livre e esclarecido (TCLE), que foi aprovado pelo comitê de ética e pesquisa da Faculdade Santa Maria. Foram excluídas as participantes que apresentaram alterações ortopédicas e posturais anteriores a gestação (escoliose e cifose) e que não aceitaram assinar o termo de consentimento livre e esclarecido.

A pesquisa foi realizada na unidade de saúde da família Amélio Cartaxo Dantas Estrela no setor obstétrico. Localizado na cidade de Cajazeiras- PB no alto sertão Paraibano. Foram incluídas na pesquisa gestantes com idade entre 16 e 39 anos e com gestação sem complicações.

A amostra foi composta por 20 gestantes, que tiveram seus dados coletados em forma de entrevista, utilizando um questionário com informações sociodemográficas e sobre queixas e desconfortos durante a gestação. Também foi feita uma avaliação postural onde observou-se a presença ou não das alterações no alinhamento postural na posição ortostática, na vista anterior, lateral direito e esquerdo e posterior, elaborado pelo próprio pesquisador.

Os dados obtidos foram analisados quantitativamente e manipulados nos programas Microsoft Office Excel 2007 e SPSS 15.0 version. A análise quanto a estatística descritiva, foi por meio do cálculo de porcentagem, freqüência, medidas de tendência central (média e mediana) e variabilidade (desvio-padrão).

\section{Resultados}

\section{Caracterização da amostra}

A amostra foi composta por 20 gestantes residentes na cidade de Cajazeiras/PB, com idades variando entre 16 e 39 anos $(M=23,85$ anos; $\mathrm{DP}=6,67 ; \mathrm{Md}=22,50)$.

O tempo de gestação das participantes equivale a uma média de 5,70 meses ( $\mathrm{DP}=1,89)$, de modo que a maioria (30\% da amostra) se encontra no oitavo mês gestacional.

Quanto a escolaridade, a maioria 35\% tinha Ensino Médio completo, 65\% eram do lar e 40\% tinham renda familiar de 1 salário mínimo, $50 \%$ era casada, $60 \%$ planejaram a gestação e não tinha filhos.

Número de consultas no pré-natal: as componentes da amostra passaram por uma média de 3,95 consultas $(\mathrm{DP}=1,43)$, durante o pré-natal.

As gestantes realizavam suas AVD'S normalmente (70\%) e não praticavam exercício físico $(95 \%)$.

Id en line Revista de Psicologia. Ano 8, No. 22, Fevereiro/2014 - ISSN 1981-1179.

Edição eletrônica em http://idonline.emnuvens.com.br/id 
Queixas, sintomas e desconfortos apresentados durante a gestação

Todas as gestantes (100\%) informaram sentir desconfortos, principalmente na região da coluna lombar $(65 \%)$ e nas pernas $(55 \%)$. Outras regiões como pelve anterior e tornozelo foram citadas por 15\%; abdômen, pés e mama por 10\%; ombro, coxa anterior, joelho, coluna torácica, pelve posterior e mão por $5 \%$.

O tipo de desconforto mais relatado pela a amostra foi dor (65\%), seguido por cãibra, sensação de peso e cansaço (30\%), queimação (20\%) e formigamento (15\%).

Em uma escala intervalar de 10 pontos, de 0 (zero) a 10 (dez), tipo Likert, para verificar o nível de desconforto entre as componentes da amostra, as participantes pontuaram entre os escores 3 (três) e 10 (dez), apresentando uma média de 5,95 ( $\mathrm{DP}=1,82 ; \mathrm{Md}=6,00)$.

O período do dia informado pelas gestantes, em que o desconforto era pior foi à noite (40\%); ao acordar (30\%); meio da tarde e igual durante todo dia (15\%); meio da manhã (10\%).

As grávidas informaram que o início do desconforto apareceu principalmente no primeiro trimestre $(60 \%) ; 80 \%$ afirmaram que o desconforto não impedia suas atividades, mas piorava a intensidade deste desconforto $(65 \%)$.

Os sintomas mais relatados pelas gestantes foram: enjôo (50\%); tontura (30\%); dificuldade de caminhar (25\%); falta de ar (15\%); edema e falta de equilíbrio (10\%).

\section{Avaliação postural das gestantes}

A postura da cabeça mostrou poucas alterações significativas, na vista anterior 5\% apresentou inclinação para a esquerda; na vista lateral evidenciou-se anteriorização (35\%) e retificação (15\%); e na vista posterior lateralização à direita $(25 \%)$.

Tabela I- Avaliação Postural da Cabeça

\begin{tabular}{lll}
\hline Postura da cabeça & Vista Anterior & \\
\hline & $F$ & $\%$ \\
Centralizada & 19 & 95 \\
Inclinada Esquerda & 1 & 5 \\
Inclinada Direita & 5 & 0 \\
Total & 20 & 100
\end{tabular}

\section{Continua...}

Id en line Revista de Psicologia. Ano 8, No. 22, Fevereiro/2014 - ISSN 1981-1179.

Edição eletrônica em http://idonline.emnuvens.com.br/id 


\begin{tabular}{lll}
\hline & Vista Lateral & \\
\hline Normal & $F$ & $\%$ \\
Anteriorizada & 10 & 50 \\
Retificada & 7 & 35 \\
Total & 3 & 15 \\
& 20 & 100 \\
\hline Centralizada & Vista Posterior & $\%$ \\
Lateralizada Direita & $F$ & 70 \\
Lateralizada Esquerda & 14 & 25 \\
Total & 5 & 5 \\
\hline
\end{tabular}

A postura da Coluna Cervical mostrou alterações na vista lateral apresentando hiperlordose $(35 \%)$ e retificação $(15 \%)$.

Tabela II: Avaliação Postural da Coluna Cervical

\begin{tabular}{lcl}
\hline Postura da Coluna Cervical & & \\
\hline & Vista Anterior & $\%$ \\
\hline Centralizada & $F$ & 100 \\
Inclinada Esquerda & 20 & 0 \\
Inclinada Direita & 0 & 0 \\
Total & 0 & 100 \\
& 20 & $\%$ \\
\hline Normal & Vista Lateral & 70 \\
Hiperlordose & $F$ & 15 \\
Retificada & 14 & 15 \\
Total & 3 & 100 \\
& 3 & $\%$ \\
& 20 & 100 \\
Centralizada & $F$ & 0 \\
Inclinada Esquerda & 20 & 0 \\
Inclinada Direita & 0 & 100 \\
\hline Total & 0 & \\
\hline
\end{tabular}

A avaliação da postura dos ombros mostrou que na vista anterior e posterior, em sua maioria o ombro esquerdo estava mais elevado (70\%). Na vista lateral $45 \%$ apresentava-se protusos. 
Tabela III- Avaliação postural dos Ombros

Postura dos ombros

\begin{tabular}{lll}
\hline & Vista Anterior & $\%$ \\
\hline Nivelados & $F$ & 25 \\
\hline Elevado Direita & 5 & 5 \\
\hline Elevado Esquerda & 1 & 70 \\
\hline Total & 14 & 100 \\
& 20 & $\%$ \\
\hline Normal & Vista Lateral & 55 \\
\hline Protusos & $F$ & 45 \\
\hline Total & 11 & 100 \\
& 9 & \\
\hline Nivelados & 20 & 25 \\
\hline Elevado Direita & Vista Posterior & 5 \\
\hline Elevado Esquerda & $F$ & 70 \\
Total & 5 & 100 \\
\hline
\end{tabular}

A avaliação postural da coluna Dorsal mostrou que na vista anterior apenas 5\% apresentava inclinação à esquerda; na vista lateral, $5 \%$ apresentavam retificação; e na vista posterior $20 \%$ tinha lateralização à esquerda e $15 \%$ lateralização à direita.

Tabela IV- Avaliação postural da Coluna Dorsal

Postura da coluna dorsal

\begin{tabular}{lll}
\hline & Vista Anterior & \\
\hline Centralizada & $F$ & $\%$ \\
\hline Inclinada Esquerda & 19 & 55 \\
\hline Inclinada Direita & 1 & 0 \\
\hline Total & 0 & 100 \\
& 20 & $\%$ \\
\hline Normal & Vista Lateral & 95 \\
\hline Retificada & $F$ & 5 \\
\hline Total & 19 & 100 \\
\hline & 1 & \\
\hline Centralizada & 20 & 6 \\
\hline Lateralizada Direita & Vista Posterior & 65 \\
\hline Lateralizada Esquerda & $F$ & 15 \\
\hline Total & 13 & 20 \\
\hline
\end{tabular}

Quanto à pelve, na vista anterior e posterior $25 \%$ tem elevação à direita e $20 \%$ elevação a esquerda; na vista lateral, $35 \%$ tem anteroversão e $15 \%$ retroversão. 
Tabela V- Avaliação postural da Pelve

\begin{tabular}{lll}
\hline Postura da Pelve & Vista Anterior & \\
\hline & $F$ & $\%$ \\
\hline Nivelados & 11 & 55 \\
Elevado Direita & 5 & 25 \\
\hline Elevado Esquerda & 4 & 20 \\
Total & 20 & 100 \\
& Vista Lateral & \\
\hline Normal & $F$ & 50 \\
Anteroversão & 10 & 35 \\
\hline Retroversão & 7 & 15 \\
Total & 3 & 100 \\
& 20 & $\%$ \\
\hline & Vista Posterior & 55 \\
Nivelados & $F$ & 25 \\
Elevado Direita & 11 & 20 \\
Elevado Esquerda & 5 & 100 \\
\hline Total & 4 & \\
\hline
\end{tabular}

Os joelhos, na vista anterior mostraram rotação interna (20\%), genovalgo (5\%) e genovaro (5\%). Na vista lateral, $10 \%$ apresentou flexão e $10 \%$ hiperextensão.

Tabela VI- Avaliação postural dos Joelhos

Postura dos Joelhos

\begin{tabular}{lll}
\hline & Vista Anterior & \\
\hline Nivelados & $F$ & 70 \\
\hline Rotação interna & 14 & 20 \\
Genovalgo & 4 & 5 \\
Genovaro & 1 & 5 \\
Total & 1 & 100 \\
& 20 & \\
\hline Normal & Vista Lateral & 80 \\
Flexão & $F$ & 10 \\
Hiperextensão & 16 & 10 \\
Total & 2 & 100 \\
\hline
\end{tabular}

As patelas em sua maioria estão medializadas (70\%) e o ângulo poplíteo encontra-se nivelado $(85 \%)$. 
Tabela VII- Avaliação postural das Patelas

\begin{tabular}{lll}
\hline Postura das Patelas & Vista Anterior & \\
\hline & $F$ & $\%$ \\
Medializadas & 14 & 70 \\
Lateralizadas & 5 & 25 \\
Elevada & 1 & 5 \\
Total & 20 & 100 \\
Angulo Poplíteo & Vista Posterior & \\
& $F$ & $\%$ \\
Nivelados & 17 & 85 \\
Elevado Direita & 3 & 15 \\
Elevado Esquerda & 0 & 0 \\
Total & 20 & 100 \\
\hline
\end{tabular}

Os pés mostraram-se em sua maioria ser do tipo cavo (80\%), mas também encontrou-se do tipo plano (15\%) e em eversão (5\%).

Tabela VIII- Avaliação postural dos Pés

\begin{tabular}{lll}
\hline Postura dos Pés & Vista Anterior & \\
\hline & $F$ & $\%$ \\
\hline Plano & 3 & 15 \\
Cavo & 16 & 80 \\
Eversão & 1 & 5 \\
Total & 20 & 100 \\
& Vista Posterior & \\
\hline Plano & $F$ & 15 \\
Cavo & 3 & 80 \\
Eversão & 16 & 5 \\
Total & 1 & 100 \\
\hline
\end{tabular}

\section{Inspeção dinâmica}

$\mathrm{Na}$ avaliação da coluna cervical, $30 \%$ das gestantes faziam flexão parcial e $20 \%$ faziam com dificuldade. No movimento de extensão, $15 \%$ faziam parcialmente e $15 \%$ com dificuldade ou dor. 
Tabela IX- Avaliação dinâmica da Coluna Cervical

\begin{tabular}{lll}
\hline \multicolumn{1}{c}{ Cervical } & Flexão & \\
\hline & $F$ & $\%$ \\
\hline Completa & 10 & 50 \\
\hline Parcial & 6 & 30 \\
Com dificuldade ou dor & 4 & 20 \\
\hline Total & 20 & 100 \\
& Extensão & $\%$ \\
\hline Completa & $F$ & 70 \\
\hline Parcial & 14 & 15 \\
\hline Com dificuldade ou dor & 3 & 15 \\
\hline \multicolumn{1}{c}{ Total } & 3 & 100 \\
\hline
\end{tabular}

Na avaliação dinâmica do tronco, $50 \%$ das gestantes faziam flexão com dificuldade e $30 \%$ faziam parcialmente. No movimento de extensão, $55 \%$ faziam parcialmente e $20 \%$ com dificuldade ou dor.

Tabela X- Avaliação dinâmica do Tronco

\begin{tabular}{lll}
\hline Tronco & Flexão & \\
\hline & $F$ & $\%$ \\
\hline Completa & 4 & 20 \\
\hline Parcial & 6 & 30 \\
Com dificuldade ou dor & 10 & 50 \\
\hline Total & 20 & 100 \\
& Extensão & $\%$ \\
\hline Completa & $F$ & 25 \\
\hline Parcial & 5 & 55 \\
\hline Com dificuldade ou dor & 11 & 20 \\
Total & 4 & 100 \\
\hline
\end{tabular}

Quanto ao movimento de báscula, 15\% não realizava ou realizava com dificuldade (15\%). Já a avaliação da musculatura abdominal mostrou que a maioria apresentava-se normal (65\%), mas também observou-se flacidez (25\%) e diástase (10\%) em algumas grávidas. 


\section{Discussão}

\section{Perfil Sócio-econômico}

Dados mostrados no estudo que avaliou o nível de atividade física habitual em gestante entre os usuários do programa saúde da família do município de Itabuna Bahia, informaram que entre os indivíduos atendidos pelas unidades a maioria eram mulheres de baixo nível socioeconômico e escolaridade (SILVA et al, 2011). No presente estudo a maioria 35\%, tinha o ensino fundamental completo e $40 \%$ tinha renda familiar de um salário mínimo, esse achado de baixo nível socioeconômico e escolaridade corroboram com a pesquisa anteriormente citada. Sabe-se, que o nível de escolaridade reflete em menores possibilidades de inserção ocupacional e participação no mercado de trabalho.

Um fato interessante é que a realidade socioeconômica parece ter influência no percentual de gestantes que apresentaram dor lombar, devido á necessidade de executar as tarefas domésticas, em razão da ausência de outra pessoa que as executasse (SILVA et al, 2011).

\section{Queixas e desconfortos durante a gestação}

Segundo Assis et al (2004), a dor lombar é uma queixa comum na população geral, considerada um sintoma freqüente e habitual nas gestantes, principalmente nos últimos meses gestação. Durante essa fase, a maioria das grávidas queixa-se de dor na coluna vertebral, especialmente na coluna lombar. O estudo mostrou que a dor lombar está presente em $65 \%$ o que condiz com dados da literatura, cuja porcentagem varia de $48 \%$ a $83 \%$.

Os resultados do presente estudo evidenciou que essa dor, seguido por câimbras, sensação de peso e cansaço (30\%), queimação (20\%) e formigamento (15\%) não teve tanta relevância quanto à dor lombar. A compressão dos grandes vasos pelo o útero gravídico, causando diminuição do fluxo sanguíneo medular, é citado como um dos fatores da lombalgia da gravidez, especialmente no último trimestre gestacional. (NORA et al, 2010). Para Sant'ana et al (2006), a etiologia da dor lombar na gravidez é mal definida, e sua origem ainda é desconhecida.

Spier (2009) em seu estudo, afirma que na gestação muitas mulheres queixam-se de dores nas mãos, sendo encontrado na presente pesquisa apenas em 5\% das mulheres, e dores em membros inferiores foram referidas no terceiro trimestre, devido à retenção de líquidos, geralmente pela diminuição da flexibilidade muscular. 
Para Souza (2009), deve-se evitar permanecer em pé por tempo prolongado, pois pode levar a algia nos pés, fato que foi encontrado no atual estudo em $10 \%$ das gestantes.

Freire (2011) mostra que o sistema gastrintestinal sofre com as alterações hormonais e também adaptações estruturais ao feto. Náuseas, vômitos, por determinados alimentos e dores abdominais são comuns durante a gravidez. Vários níveis de náuseas e vômitos, chamados de náuseas matinais, aparecem em $50 \%$ a $90 \%$ das mulheres durante o primeiro trimestre; concordando com o autor, náuseas foi citado na atual pesquisa por $50 \%$ das participantes.

No sistema respiratório o consumo de oxigênio aumenta 14\% resultando no aumento da função cardíaca e na frequência respiratória. Os níveis de progesterona altos causam hiperventilação e diminuição de dióxido de carbono nos alvéolos, favorecendo a difusão de dióxido de carbono da circulação fetal para a materna. Com isso ocorre à falta de ar, que foi relatada por $15 \%$ das participantes da pesquisa (ASSIS, 2004).

Um fato importante descrito pelas gestantes da pesquisa é que $80 \%$ relatavam ter algum desconforto, que não impedia a realização, porém, intensificava-se durante as AVD’s (65\%).

\section{Atividade física durante a gestação}

O presente estudo constatou que $95 \%$ das participantes não realizavam exercícios físicos, fato não positivo, pois segundo Mann et al (2008), o exercício físico ajudaria para uma melhora na adaptação a nova postura física, contribuindo para uma melhor habilidade da gestante durante à prática do trabalho diário.

O fisioterapeuta, por meios de orientações posturais e exercícios, tem como objetivos prevenir, lesões musculares, contando com atividades que propiciam as gestantes pensar e viver um corpo em alterações, procurando modificar as regras que inibem a consciência corporal através da reeducação postural. Para conseguir uma postura adequada durante a gravidez, sugerindo algumas orientações básicas para que a gestante tenha um máximo de conforto possível e um bom desempenho nas suas atividades de vida diária. (FREITAS, 2008).

Os benefícios da prática de atividades físicas durante a gestação são vários e atingem diferentes áreas do organismo materno. O exercício diminui e previne as lombalgias, devido à orientação da postura correta da gestante frente à hiperlordose que comumente surge durante a gestação, em função da expansão do útero na cavidade abdominal e o conseqüente desvio do centro gravitacional. Nestes casos, o exercício físico ajudará para adaptação de nova postura física, contribuindo em maior habilidade para a gestante durante a prática da atividade física e do trabalho diário (BATISTA, 2003). 


\section{Alterações posturais das gestantes}

Nora (2010) define postura como sendo um conjunto de posições de todas as articulação do corpo no determinado momento, e ainda refere que o alinhamento esquelético ideal envolve uma quantidade mínima de estresse e tensão e é favorável a eficiência máxima do corpo. Na grávida observam-se os ajustes através da pélvis anterior ou do aumento ou diminuição da curvatura lombar.

A adaptação lombar pode ser pela diminuição da ação do músculo íliopsoas, já que seu torque de flexão não tem mais utilidade, uma vez que o peso do feto realiza essa função (ASSIS, 2004).

As mudanças que ocorrem no corpo feminino acontecem devido à quantidade excessiva de hormônios, responsáveis pelas adaptações do organismo a sua nova condição. As alterações acontecem principalmente nos sistemas cardiorrespiratório, musculoesquelético e no metabolismo geral; não se restringindo apenas aos órgãos, mas também a mecânica do corpo feminino, tais como alterações no centro de gravidade, na postura e no equilíbrio (SANTOS, 2010).

Foi observado nos estudo de Ribas e Guirro (2005), que a alteração da distribuição do peso na região plantar dos pés e o aumento da oscilação anteroposterior do corpo, devido à ajustes posturais, pode provocar a anteriorização da cabeça das gestantes, o que foi comprovado na presente pesquisa, sendo evidenciado em 35\% das grávidas, além de intensificação da hiperextensão da coluna cervical e lombar levando a hiperlordose cervical e aumento da extensão dos joelhos e tornozelo, para conseguir manter o equilíbrio postural, fato que corrobora com o autor supracitado.

Segundo Baracho (2002) as mudanças posturais se tornam mais evidentes após a $20^{\circ}$ semana de gestação, quando será percebido o crescimento abdominal e das mamas. O deslocamento do centro de gravidade pra frente e para cima é consequiência do alargamento do útero e das mamas. A mudança do centro de gravidade leva o organismo a compensar com a postura para dar equilíbrio e estabilidade (LEMOS et al, 2009)

Ocorrem ainda outras modificações posturais, como protusão dos ombros que foi notado no atual estudo na vista lateral, aparecendo em $45 \%$ das grávidas corroborando com o autor.

Na vista anterior encontrou-se elevação do ombro à direita em $70 \%$ das pesquisadas. Durante a gestação, ocorrem mudanças na mecânica do esqueleto da gestante devido à ação de hormônios que causam frouxidão ligamentar, surgindo mudanças biomecânicas que provocam modificações na estática e dinâmica do esqueleto.

A ação hormonal decorrente principalmente do hormônio relaxina, que provoca aumento do relaxamento articular e ligamentar, levando a instabilidade articular, normalmente favorece uma anteroversão pélvica, estando presente na atual pesquisa em $35 \%$ das gestantes fato que favoreceu o surgimento da hiperlordose lombar (NORA, 2010). 
Spier (2009), observou que 41,7\% das gestantes tinham joelhos varos, 58,3\% valgos e 88,3\% hiperextendido, números que não foram semelhantes a atual pesquisa, pois $80 \%$ dos joelhos avaliados eram normais e apenas $10 \%$ hiperextendidos.

Considerando-se joelho hiperextendido, aquele que tem o um ângulo menor que 170 graus, e joelho valgo aquele que os côndilos se tocam e os maléolos não, e varo quando os maléolos se tocam e os joelhos não (SANTOS, 2010)

Quanto à rotação interna do joelho foi encontrado em $20 \%$ das pesquisadas, fato contrário ao encontrado na pesquisa de Senhorinho et al (2003), que detectou rotação externa em $10 \%$ de sua amostra.

Spier (2009) em sua pesquisa detectou que 75\% das gestantes apresentaram patelas medializadas e $70 \%$ elevadas; a causa dessa alteração no caso da patela medializada, pode estar relacionado ao encurtamento do músculo vasto medial obliquo ou a fraqueza muscular do vasto lateral, corroborando com o autor acima, o presente estudo encontrou $70 \%$ de patelas medializadas.

Os pés são a principal fonte de sustentação do ser humano, distribuindo entre eles o peso de acordo com as necessidades mecânicas adaptação postural em toda planta do pé. O aumento do peso corporal durante a gestação faz com que a mulher aumente sua base de sustentação, o que é conseguido pelo o afastamento dos pés e o desabamento do arco longitudinal. Este fato não foi encontrado na pesquisa atual, pois em sua maioria as grávidas apresentaram pés cavos em $80 \%$ e planos em apenas $15 \%$, enquanto que na pesquisa de Spier (2009) foram registrados pés planos em $44 \%$ das gestantes.

Os pés planos ocorrem principalmente por uma insuficiência muscular do tibial posterior, ou mais freqüentemente, do fibular lateral longo, no momento em que o peso do corpo se descarrega sobre o arco longitudinal e este desaba (SPIER, 2009).

\section{Postura e gravidez}

Para Spier ((2009), muitas mulheres ao engravidar desconhecem o funcionamento interno do seu próprio corpo. A consciência dos efeitos é importante nas mudanças e suas alterações sobre a postura. Nessa fase uma postura correta deve ser dinâmica e vital, variando sempre com suas necessidades.

A gravidez envolve várias alterações, em todo corpo inclusive nos músculos, articulações e nos ossos. À medida que o útero aumenta de tamanho o centro de gravidade da gestante tende a alterar-se, forçando-a a adaptar-se. Muitas adotam posturas incorretas, colocando a coluna vertebral e 
articulações um esforço desnecessário. Nesta fase além da gestante tomar consciência do próprio corpo e reeducar sua postura.

Fator que é necessário considerar é que na maioria das atividades se utiliza mais o membro superior, cabeça e tronco. Assim a inclinação do corpo, para a realização de determinadas tarefas poderá sobrecarregar os músculos, tendões e articulações, devendo considerar além deste fator, o peso adquirido em função da gravidez. A gestante para inclinar ou curvar seu tronco encontrará dificuldade pelo volume do abdômen, provocando ou dificultando sua respiração. Foi visto no presente estudo que ao realizar a flexão do tronco 50\% das participantes referiram dificuldade ou dor (FREITAS, 2008).

Para Silva (2011), durante a gravidez a musculatura abdominal é indispensável para permitir o crescimento uterino, ocorrendo uma separação dos feixes dos músculos retos abdominais. São considerados fatores predisponentes para diástase dos músculos retos abdominais, a obesidade, as gestações múltiplas e multiparidade, a macrossomia fetal e a flacidez da musculatura abdominal e prégravídica, levando uma maior distensão abdominal durante a gravidez. Essa condição não foi representativa na presente pesquisa visto que $65 \%$ das grávidas apresentaram firmeza na musculatura do abdômen e apenas $10 \%$ tiveram diástase.

\section{Conclusão}

O estudo evidenciou que a lombalgia gestacional é a queixa mais freqüente em gestantes, visto que a maioria das participantes referiram sentir este desconforto. Quanto às alterações posturais, foi encontrado principalmente anteriorização da cabeça, protusão de ombros, hiperlordose lombar e anteroversão pélvica.

Esperamos que o presente estudo possa contribuir para a descrição, compreensão e reflexões mais embasadas sobre as questões relacionadas a postura e desconfortos das gestantes, servindo de apoio para outros estudiosos sobre o tema e, para embasar políticas públicas sobre a saúde da mulher gestante, ajudando a prevenir esse tipo de problema de saúde.

\section{Referências}

ASSIS, R. G. A. et al; Prevalência e características da lombalgia na gestação: um estudo entre gestantes assistidas no programa de pré-natal da maternidade dona íris em Goiânia [Monografia]. Universidade Católica de Goiás. Goiânia - GO, 2004 
BATISTA, D. C. et al; Atividade física e gestação: saúde da gestante não atleta e crescimento fetal.

Rev. Bras. Saude Mater. Infant. vol. 3 nº 2 Recife Apr./June 2003

BRASIL, Ministério da Saúde, Conselho Nacional de Saúde. Comissão Nacional de Ética em Pesquisa. Resolução 196/1996 sobre pesquisas envolvendo seres humanos. Brasília: 1996.

FREIRE, R. M. G.; Os efeitos da fisioterapia em meio aquático nas grávidas [Monografia]. Universidade Atlântica. Barcarena, Pará - PA, 2011

FREITAS, R. S.; Considerações ergonômicas no período gravídico e puerperal [Monografia] Universidade Veiga de Almeida. Rio de Janeiro - RJ, 2008.

LEMOS, L. F. C. et al; Uma revisão sobre centro de gravidade e equilíbrio corporal. R. bras. Ci. e Mov 2009;17(4):83-90.

MACHADO, B. L. R. el al.; Incidência de dor lombar em gestantes. [Monografia] Centro Universitário Franciscano - UNIFRA. Santa Maria - RS, 2011.

MANN, L. et al; Dor lombo-pélvica e exercício físico durante a gestação. Fisioter. Mov. 2008 abr/jun;21(2):99-105

MARTINS, Junior. Como escrever trabalhos de conclusão de curso. 2 ed. Rio de Janeiro: 2008.

MOREIRA, L. S. et al; Alterações posturais, de equilíbrio e dor lombar no período gestacional. FEMINA, Maio 2011, vol 39, nº 5 .

NORA, D. et al; Alterações Posturais em gestantes e suas influências na biomecânica da coluna. [Monografia]. Universidade Federal Santa Maria. Rio Grande do Sul - RS, 2010.

SANT'ANA, P. F. et al. Caracterização da dor lombar em gestantes atendidas no Hospital Universitário de Brasília. Ciências da Saúde, 2006, vol 4, nº 1 / 2, p. 37-48. 
SANTOS, M. M. et al; Lombalgia gestacional: prevalência e características de um programa pré-natal. Arq Bras Ciên Saúde, Santo André, vol 35, nº 3, p 147-9, 2010

SENHORINHO, H. C. et al; Alterações fisiopatológicas no período gestacional, relacionada à ocupação das gestantes do "lar preservação da vida" no município de Maringá no ano de 2002. Iniciação Cientifica Cesumar, jan-jun 2003, vol 05, nº 01, p 13-22.

SILVA, K. B. et al; Prevalência da lombalgia e sua associação com atividade doméstica em gestantes do município de Itabuna, Bahia. Revista Brasileira de Saúde Pública, abr-jun 2011, vol 35, nº 2, p 387-396.

SOARES, A. P. et al; Avaliação da atuação fisioterapêutica em obstetrícia sob a ótica de médicos e fisioterapeutas nos hospitais do município de Vila Velha-ES. [Monografia] Faculdade Novo Milênio. Vila Velha - ES, 2008.

SOUZA, P. Os benefícios da fisioterapia nas alterações posturais em grávidas do $3^{\circ}$ ao $9^{\circ}$ mês. [Monografia] Faculdade Ávila.Goiânia - GO, 2009.

SPIER, M. As alterações musculoesqueléticas e as queixas de dor e desconforto em gestantes adolescentes [Monografia] Centro Universitário Feevale. Novo Hamburgo, 2009.

Como citar este artigo (Formato ISO):

SANTANA, F.M.; SILVA, F.G.; CARVALHO, D.C.S.N.; DENOA, D.L.B.; LINS, H.L.C.C. MARTINS, M.E.P. Análise da Postura e dos Desconfortos de Gestantes atendidas pela Estratégia Saúde da Família. Id on Line Revista de Psicologia, Fevereiro de 2014, vol.8, n.22, p. 33-48. ISSN 1981-1189. 